\title{
Recent Femtoscopy Results from NA49: Evidence for a Non-Gaussian Tail in the 3-Dimensional Two-Pion Emission Source at SPS
}

\author{
P. Chung ${ }^{1}$ and P. Danielewicz ${ }^{2}$ for The NA49 Collaboration \\ ${ }^{1}$ Dept of Chemistry, SUNY Stony Brook, Stony Brook, NY 11794, USA \\ ${ }^{2}$ National Superconducting Cyclotron Laboratory and Department of Physics and Astronomy, \\ Michigan State University, East Lansing, MI 48824-1321, USA
}

\begin{abstract}
The NA49 experiment at CERN SPS has acquired a huge data set of $\mathrm{Pb}+\mathrm{Pb}$ events over a broad range of energy and centrality during the last several years. This high statistics data set, coupled with a state-of-the-art analysis technique, allows for the first model-independent extraction and energy scan of 3D emission sources for pion pairs at SPS energies. These 3D two-pion emission sources provide new insights into the nature of a long-range source previously reported by PHENIX at RHIC. The new results indicate that the two-pion source function is essentially Gaussian from $20 \mathrm{AGeV}$ to $80 \mathrm{AGeV}$ but it displays significant non-Gaussian tails at 158 AGeV.
\end{abstract}

Keywords: Correlation; Interferometry; Intensity interferometry; Cartesian harmonics; Correlation anisotropy

\section{INTRODUCTION}

A deconfined phase of nuclear matter is expected to be formed at the high energy densities created in relativistic heavy ion collisions [1]. It is widely believed that important signatures of such a phase are reflected in the space-time extent and shape of particle emission source functions.

Recently, 1-Dimensional source imaging techniques [2-4] have revealed a non-trivial long range structure in the twopion emission source at RHIC [5, 6]. The origins of this structure are still unclear. The presence/absence of such a structure in the pion emission sources in heavy ion collisions at intermediate SPS energies could yield important information which could help resolve the structure's origins. The NA49 Collaboration has carried out $\mathrm{Pb}+\mathrm{Pb}$ collisions over a wide range of bombarding energies at the CERN SPS during the last decade [7]. Such a rich data set provides a unique opportunity to search for this long range structure at the SPS and study its evolution with beam energy, and hopefully, unravel its nature.

In this paper, the 3-Dimensional emission source images for pions produced in central $\mathrm{Pb}+\mathrm{Pb}$ collisions over the incident energy range 20 to $158 \mathrm{AGeV}$ are presented. The results are discussed in the context of a Gaussian shape assumption.

\section{EXPERIMENTAL SETUP}

The data presented here were taken by the NA49 Collaboration during the years 1996-2002. The incident beams of 20, 40, 80, $158 \mathrm{AGeV}$ were provided by the CERN SPS accelerator. The NA49 Large Acceptance Hadron Detector [8] achieves large acceptance precision tracking $\left(\delta p / p^{2} \approx\right.$ $\left.(0.3-7) \cdot 10^{-4}(\mathrm{GeV} / \mathrm{c})^{-1}\right)$ and particle identification using time projection chambers (TPC's). Charged particles are detected by the tracks left in the TPC's and identified by the energy deposited in the TPC gas. Mid-rapidity particle identification is further enhanced by a time-of-flight wall (resolution $60 \mathrm{ps}$ ). Event centrality is determined by a forward calorimeter which measures the energy of spectator matter.

\section{DATA ANALYSIS}

3D correlation functions, $\mathrm{C}(\mathbf{q})$, were obtained as the ratio of pair to uncorrelated reference distributions in relative momentum $\mathbf{q}$ for $\pi^{-} \pi^{-}$pairs. Here, $\mathbf{q}=\frac{\left(\mathbf{p}_{1}-\mathbf{p}_{2}\right)}{2}$ is half of the relative momentum between the two particles in the Pair Center-ofMass System (PCMS) frame. The pair distribution was obtained using pairs of particles from the same event and the uncorrelated distribution was obtained by pairing particles from different events. The Lorentz transformation of $\mathbf{q}$ from the laboratory frame to the PCMS is done by a Lorentz transformation to the pair Locally Co-Moving System (LCMS) frame along the beam direction followed by a Lorentz transformation to the PCMS along the direction of the transverse momentum of the pair.

Track merging and splitting effects were removed by appropriate cuts on both the pair and uncorrelated distributions. The pair cuts require the two particles in each pair to be separated by at least $2.2 \mathrm{~cm}$ over 50 pad rows in the main vertex TPC. A $20 \%$ increase in this minimum separation results in the correlation data points fluctuating within the statistical errors. Hence, the systematic uncertainty associated with the pair cuts is deemed smaller than the statistical uncertainty.

The effects of track momentum resolution were assessed by jittering the momentum of the tracks in the data by the maximum momentum resolution, $\delta p / p^{2} \approx 7.10^{-4}(\mathrm{GeV} / \mathrm{c})^{-1}$, and re-computing the $3 \mathrm{D}$ correlation function. The resulting correlation function incorporates twice the effect of the momentum resolution and was found to be identical to the raw unsmeared correlation function. This is not surprising, considering that the mean momentum of the tracks used in this analysis is $1.2 \mathrm{GeV} / \mathrm{c}$, resulting in a momentum resolution of $\delta p / p \approx 0.1 \%$. Such a small momentum resolution has no observable effect on the correlation function.

At 40 and $158 \mathrm{AGeV}$, data were taken with positive and negative magnetic fields, in order to check the stability of the physics results and exclude non-physical contributions. The results for the two different magnetic field orientations were compared and found to be in good agreement. Hence, at both 40 and $158 \mathrm{AGeV}$, the data for positive and negative magnetic 
fields were combined in order to enhance statistics.

\section{A. Moment Calculation}

In the cartesian harmonic decomposition technique [9, 10], the $3 \mathrm{D}$ correlation function is expressed as

$$
C(\mathbf{q})-1=R(\mathbf{q})=\sum_{l} \sum_{\alpha_{1} \ldots \alpha_{l}} R_{\alpha_{1} \ldots \alpha_{l}}^{l}(q) A_{\alpha_{1} \ldots \alpha_{l}}^{l}\left(\Omega_{\mathbf{q}}\right)
$$

where $l=0,1,2, \ldots, \alpha_{i}=x, y$ or $z, A_{\alpha_{1} \ldots \alpha_{l}}^{l}\left(\Omega_{\mathbf{q}}\right)$ are cartesian harmonic basis elements ( $\Omega_{\mathbf{q}}$ is solid angle in $\mathbf{q}$ space) and $R_{\alpha_{1} \ldots \alpha_{l}}^{l}(q)$ are cartesian correlation moments given by

$$
R_{\alpha_{1} \ldots \alpha_{l}}^{l}(q)=\frac{(2 l+1) ! !}{l !} \int \frac{d \Omega_{\mathbf{q}}}{4 \pi} A_{\alpha_{1} \ldots \alpha_{l}}^{l}\left(\Omega_{\mathbf{q}}\right) R(\mathbf{q})
$$

The coordinate axes are oriented so that $\mathrm{z}$ is parallel to the beam (long) direction, $x$ points in the outward (out) direction of the total momentum of the pair in the LCMS frame and $y$ is perpendicular to the other two axes (side direction).

The correlation moments, for each order $l$, can be calculated from the measured 3D correlation function using equation (2). Alternatively, equation (1) can be truncated so as to include all non-vanishing moments and expressed in terms of independent moments only. Up to order $l=4$, there are 6 independent moments: $R^{0}, R_{x 2}^{2}, R_{y 2}^{2}, R_{x 4}^{4}, R_{y 4}^{4}$ and $R_{x 2 y 2}^{4}$ where $R_{x 2}^{2}$ is shorthand for $R_{x x}^{2}$ etc. The independent moments can then be extracted as a function of $q$ by fitting the truncated series to the experimental 3D correlation function with the moments as the parameters of the fit. In this analysis, the moments are computed up to order $1=4$ : higher order moments are either zero or negligible.

Fig. 1 shows a comparison between the 1D correlation function, $C(q)$, and the $l=0$ moment $R^{0}(q)+1$ for mid-rapidity $\left(\left|y_{L}-y_{0}\right|<0.35\right.$, where $y_{L}$ is the particle laboratory rapidity and $y_{0}$ is the CM rapidity), low $p_{T}\left(0.0<p_{T}<0.2 \mathrm{GeV} / \mathrm{c}\right)$ $\pi^{-} \pi^{-}$pairs from $20 \mathrm{AGeV}$ central $(7 \%) \mathrm{Pb}+\mathrm{Pb}$ collisions as a function of the relative momentum $q$ in the PCMS frame. The 1D correlation function is in good agreement with the $l=0$ moment $R^{0}(q)+1$, as expected in the absence of any significant angular acceptance issues, and attests to the reliability of the moment calculation technique used in this analysis. Similarly good agreement is found for 40,80 and $158 \mathrm{AGeV}$ $\mathrm{Pb}+\mathrm{Pb}$ collisions as shown in Figs. 2, 3 and 4 respectively.

\section{B. Moment Fitting}

In order to extract the source function from the 3D correlation function, it is possible to fit the latter directly with an assumed shape for the source function. Since the 3D correlation function is represented by the cartesian moments in the cartesian harmonic decomposition, this amounts to fitting the six independent moments with a trial source function. The simplest source function is the 3D Gaussian, frequently termed ellipsoid, shape.

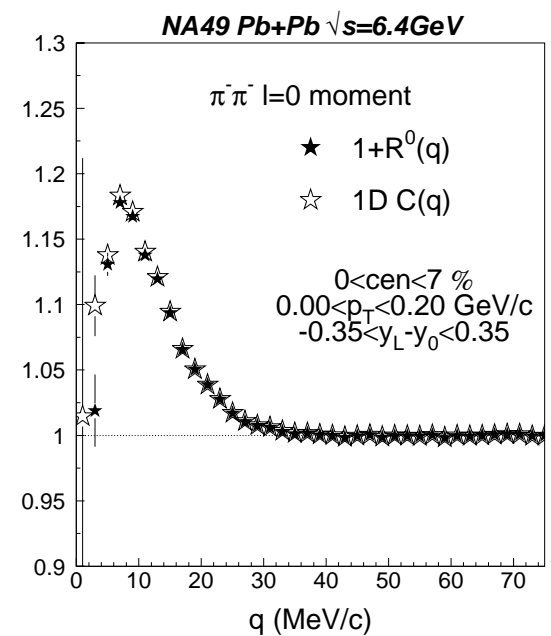

FIG. 1: 1D correlation function C(q) (open stars) and $l=0$ moment $R^{0}(q)+1$ (solid stars) for low $p_{T}$ mid-rapidity $\pi^{-} \pi^{-}$pairs from central $\mathrm{Pb}+\mathrm{Pb}$ collisions at $\sqrt{s}=6.4 \mathrm{AGeV}$ (incident beam energy of 20 $\mathrm{AGeV})$.

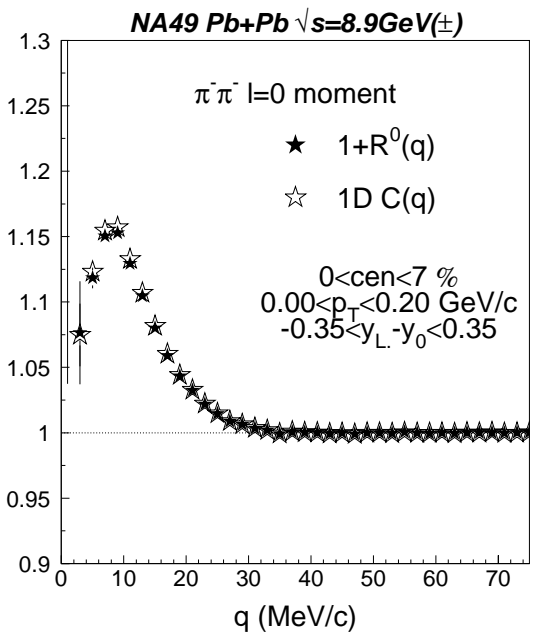

FIG. 2: Same as Fig. 1 but for $\sqrt{s}=8.9 \mathrm{AGeV}$ (incident beam energy of $40 \mathrm{AGeV})$. The result is averaged over runs with +ve and -ve magnetic field.

Fig. 5 shows the six independent moments for mid-rapidity $\left(\left|y_{L}-y_{0}\right|<0.35\right.$, low $p_{T}\left(0.0<p_{T}<0.2 \mathrm{GeV} / \mathrm{c}\right) \pi^{-} \pi^{-}$pairs from $20 \mathrm{AGeV}$ central $(7 \%) \mathrm{Pb}+\mathrm{Pb}$ collisions as a function of the relative momentum $q$ in the PCMS frame. The data points are shown in solid circles and the open squares represent the result of a simultaneous fit to the independent moments with an ellipsoid shape. The fit parameters are shown in panel (c). The data moments are fairly well represented by the Gaussian shape except for some deviation in $R_{y 2}^{2}$.

Figs. 6 and 7 show the corresponding moments for $\pi^{-} \pi^{-}$ 


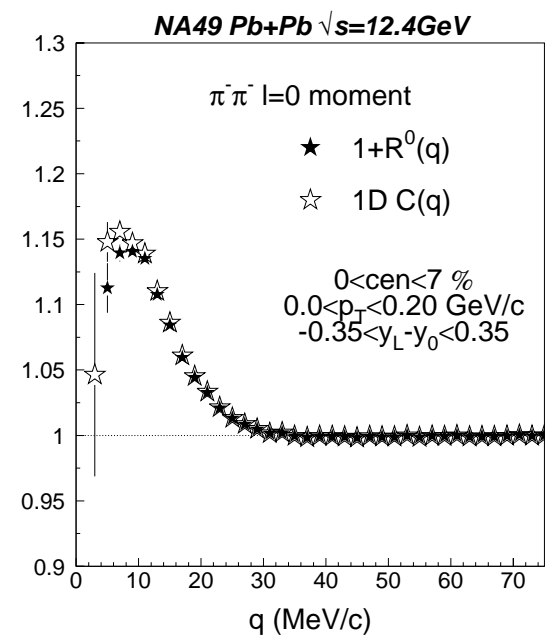

FIG. 3: Same as Fig. 1 but for $\sqrt{s}=12.4 \mathrm{AGeV}$ (incident beam energy of $80 \mathrm{AGeV}$ ).

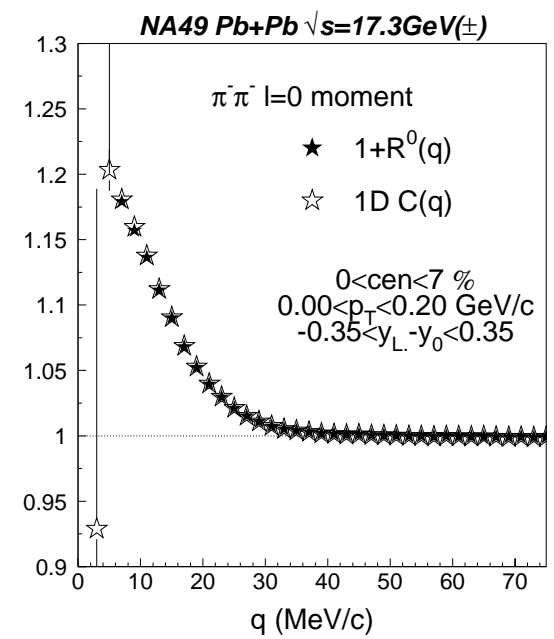

FIG. 4: Same as Fig. 1 but for $\sqrt{s}=17.3 \mathrm{AGeV}$ (incident beam energy of $160 \mathrm{AGeV}$ ). Result is average over runs with +ve and -ve magnetic field.

pairs from $40 \mathrm{AGeV}$ and $80 \mathrm{AGeV} \mathrm{Pb}+\mathrm{Pb}$ collisions respectively. In both cases, the moments display the same pattern as for $20 \mathrm{AGeV}$ and the ellipsoid fit describes most of the moments except in the $q<20 \mathrm{MeV}$ region in $R_{y 2}^{2}$.

Fig. 8 shows the corresponding moments for $\pi^{-} \pi^{-}$pairs from $158 \mathrm{AGeV} \mathrm{Pb}+\mathrm{Pb}$ collisions. In this case, the fit result shows that the data moments are not adequately described by the Gaussian assumption: $R^{0}, R_{y 2}^{2}$ and $R_{y 4}^{4}$ exhibit significant discrepancies between the data and the fit moments.
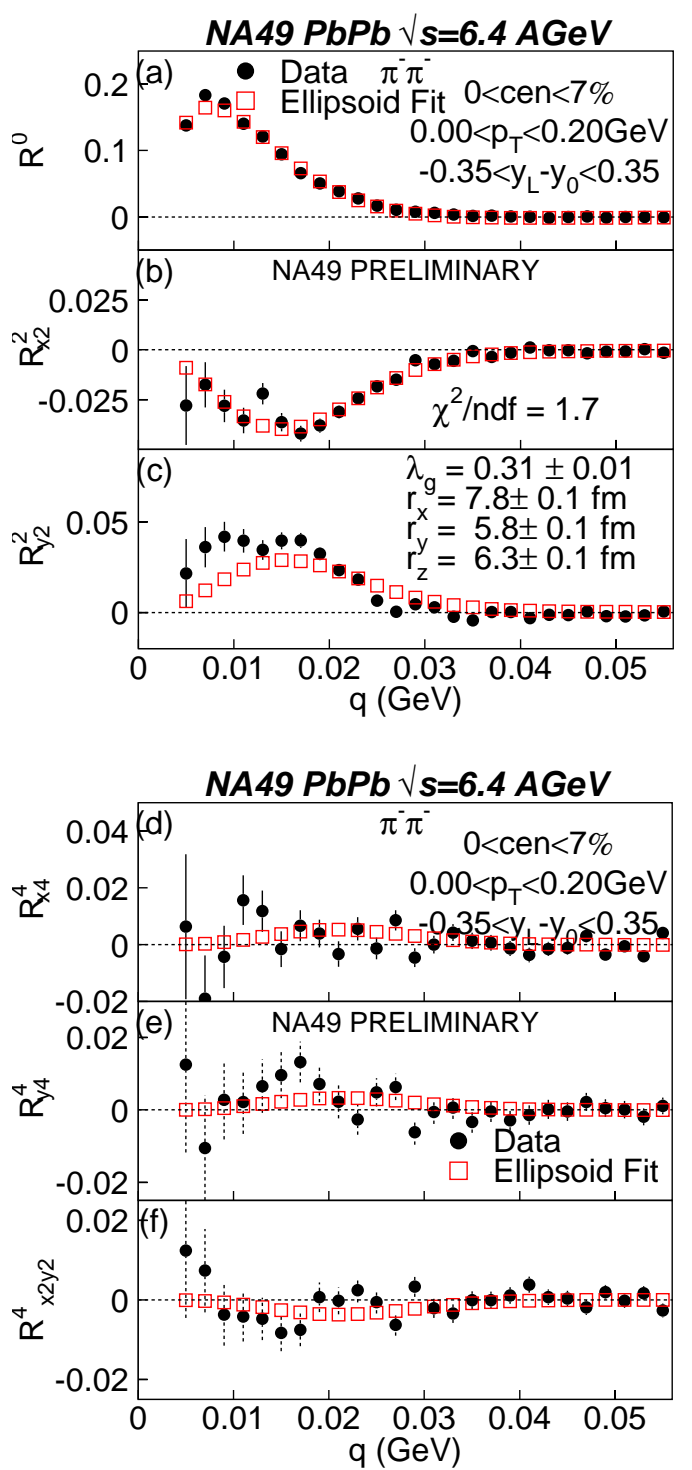

FIG. 5: (Color online) (a) $l=0, l=2((\mathrm{~b})$ and (c)) and $l=4((\mathrm{~d})$, (e) and (f)) moments for mid-rapidity low $p_{T} \pi^{-} \pi^{-}$pairs from $20 \mathrm{AGeV}$ central $\mathrm{Pb}+\mathrm{Pb}$ collisions as a function $\mathrm{q}$. Data are shown as solid circles while the squares represent the result of a simultaneous fit of the moments with an ellipsoidal shape.

\section{Moment Imaging}

Fig. 8 shows that the two-pion source function at $158 \mathrm{AGeV}$ exhibits appreciable non-Gaussian characteristics. The shape of the source can be accessed through the Source Imaging technique developed by Brown and Danielewicz [2-4].

The two-particle 1D correlation and source function are related through the Koonin-Pratt [11] equation:

$$
C(q)-1=4 \pi \int d r r^{2} K_{0}(q, r) S(r) .
$$

This is a linear integral equation that may be inverted numer- 

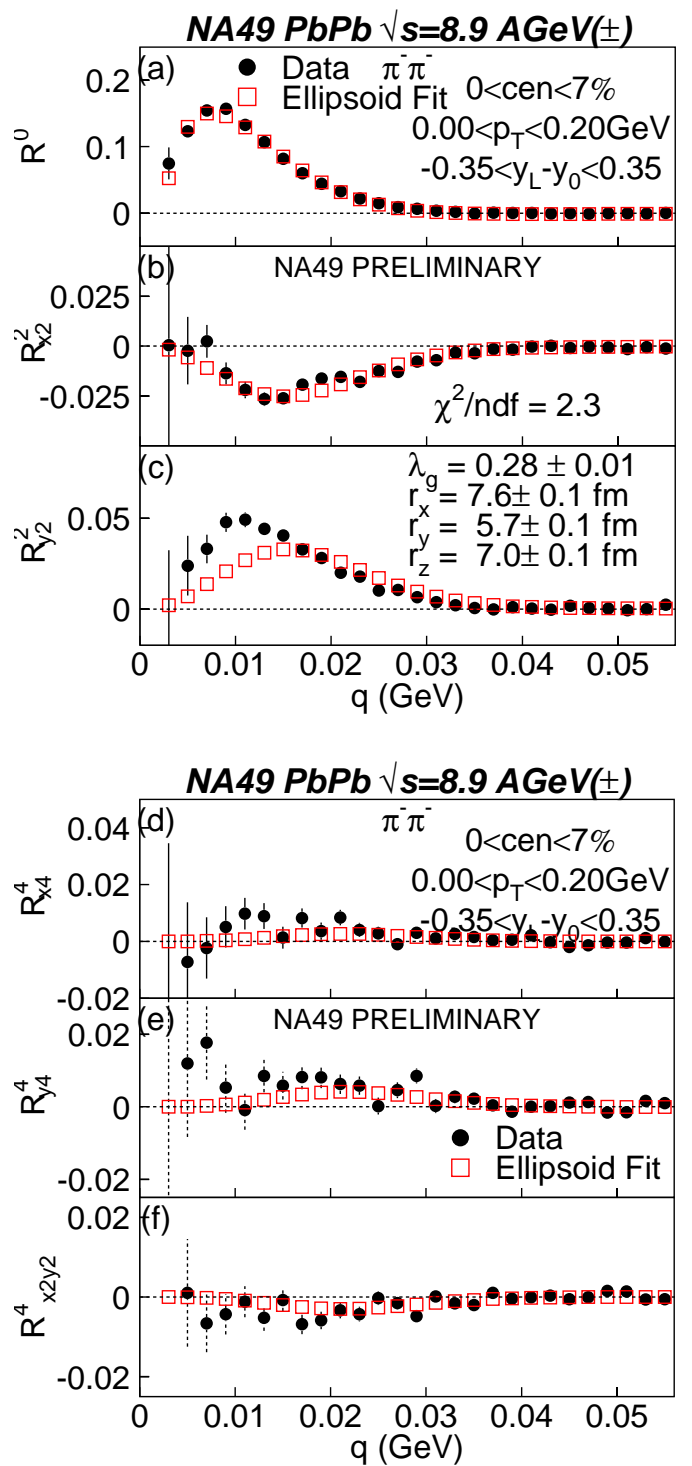

FIG. 6: (Color online) Same as Fig. 5 but for $40 \mathrm{AGeV} \mathrm{Pb}+\mathrm{Pb}$ collisions.

ically to obtain the $1 \mathrm{D}$ source function $S(r)$ using the techniques in Ref. [2-4]. Here, the imaged source function $S(r)$ gives the probability of emitting a pair of particles a distance $r$ apart in the PCMS frame.

In order to access the $3 \mathrm{D}$ source function $\mathrm{S}(\mathbf{r})$, both the $3 \mathrm{D}$ correlation function $\mathrm{C}(\mathbf{q})$ and source function $\mathrm{S}(\mathbf{r})$ are expanded in a series of the cartesian harmonic basis with correlation moments $R_{\alpha_{1} \ldots \alpha_{l}}^{l}(q)$ and source moments $S_{\alpha_{1} \ldots \alpha_{l}}^{l}(q)$ respectively. Substitution into the 3D Koonin-Pratt equation

$$
C(\mathbf{q})-1=\int d \mathbf{r} K(\mathbf{q}, \mathbf{r}) S(\mathbf{r})
$$

results [9] in a relationship, equation (5), between the correlation moments $R_{\alpha_{1} \ldots \alpha_{l}}^{l}(q)$ and source moments $S_{\alpha_{1} \ldots \alpha_{l}}^{l}(q)$ which is similar to the 1D Koonin-Pratt equation:
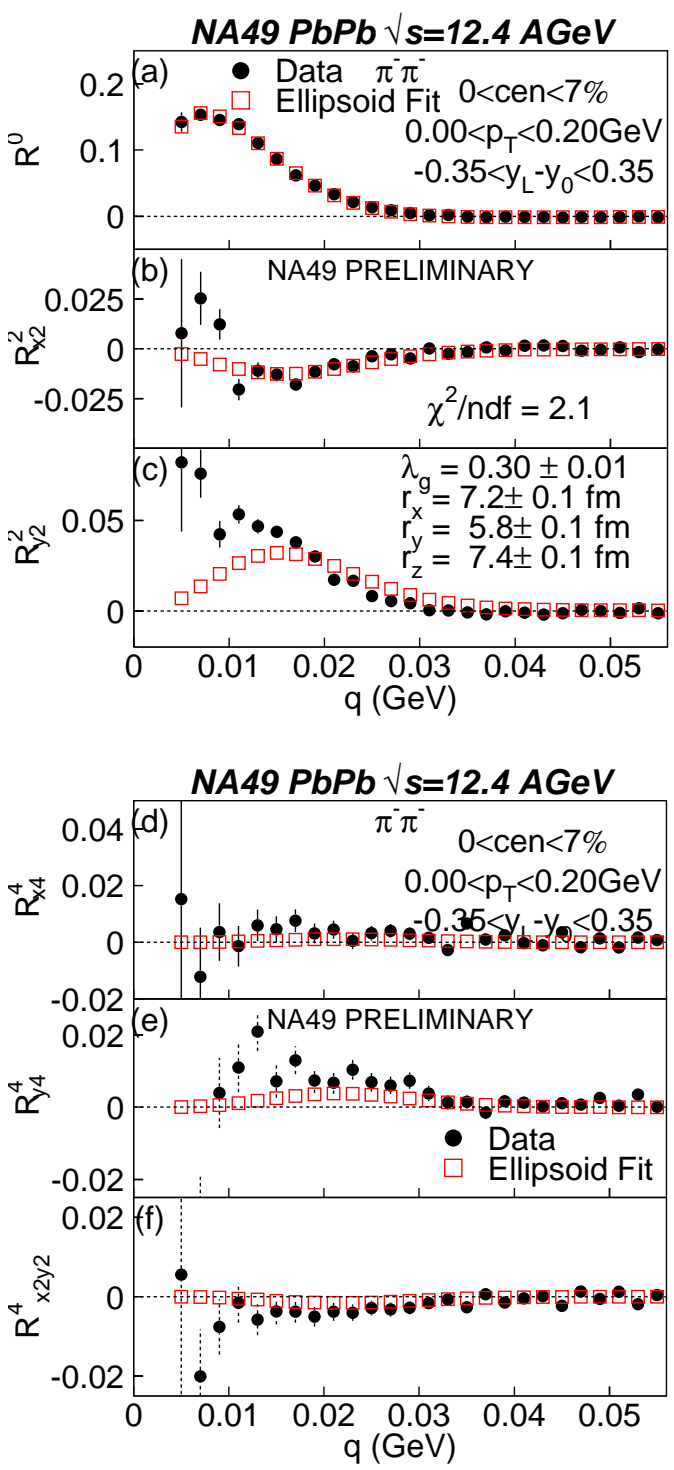

FIG. 7: (Color online) Same as Fig. 5 but for $80 \mathrm{AGeV} \mathrm{Pb+Pb} \mathrm{colli-}$ sions.

$$
R_{\alpha_{1} \ldots \alpha_{l}}^{l}(q)=4 \pi \int d r r^{2} K_{l}(q, r) S_{\alpha_{1} \ldots \alpha_{l}}^{l}(q)
$$

but now pertains to moments describing different ranks of the angular anisotropy $l$. Since the mathematical structure of Eq.5 is the same as that of 3, the same 1D Imaging technique can be used to invert each correlation moment $R_{\alpha_{1} \ldots \alpha_{l}}^{l}(q)$ to extract the corresponding source moment $S_{\alpha_{1} \ldots \alpha_{l}}^{l}(q)$. Thereafter, the total 3D source function is calculated by combining the source moments for each $l$ as in equation (6)

$$
S(\mathbf{r})=\sum_{l} \sum_{\alpha_{1} \ldots \alpha_{l}} S_{\alpha_{1} \ldots \alpha_{l}}^{l}(r) A_{\alpha_{1} \ldots \alpha_{l}}^{l}\left(\Omega_{\mathbf{r}}\right)
$$



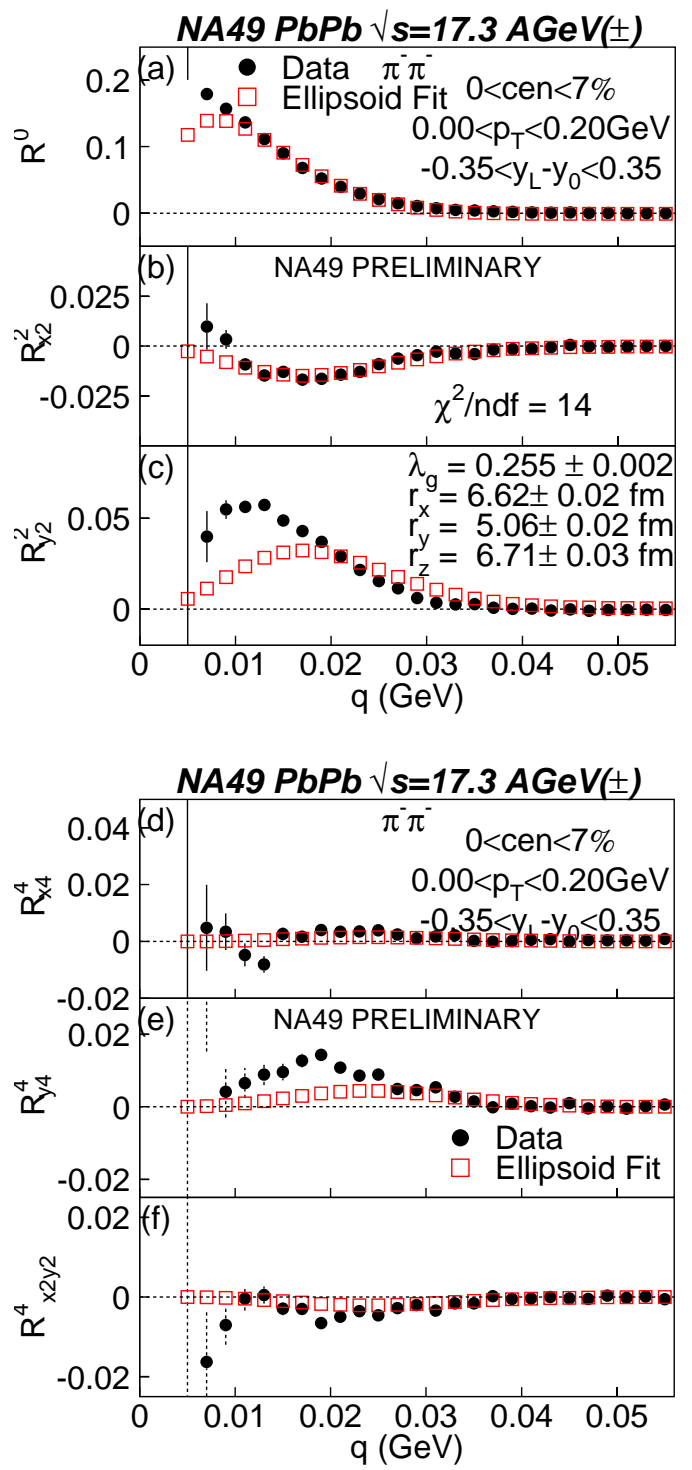

FIG. 8: (Color online) Same as Fig. 5 but for $158 \mathrm{AGeV} \mathrm{Pb}+\mathrm{Pb}$ collisions.

Bottom panel of Fig. 9 shows the imaged $l=0$ moment $S^{0}$ for the input $1=0$ moment $C^{0}(q)=R^{0}(q)+1$ for midrapidity low $p_{T} \pi^{-} \pi^{-}$pairs from $20 \mathrm{AGeV}$ central $\mathrm{Pb}+\mathrm{Pb}$ collisions. The source image looks essentially Gaussian-like. Inserting the extracted image into the 1D Koonin-Pratt equation (3) yields the restored moment shown in open squares in the top panel. The good agreement between the input and restored correlation moments serves as a consistency check of the imaging procedure.

Fig. 10 shows the corresponding extraction of the $l=0$ source moment for pions from $158 \mathrm{AGeV}$ central $\mathrm{Pb}+\mathrm{Pb}$ collisions. In contrast to the $20 \mathrm{AGeV}$ case, the $S^{0}$ moment at $158 \mathrm{AGeV}$ displays an exponential-like tail which extends beyond the Gaussian-like tail at $20 \mathrm{AGeV}$. The difference in $S^{0}$
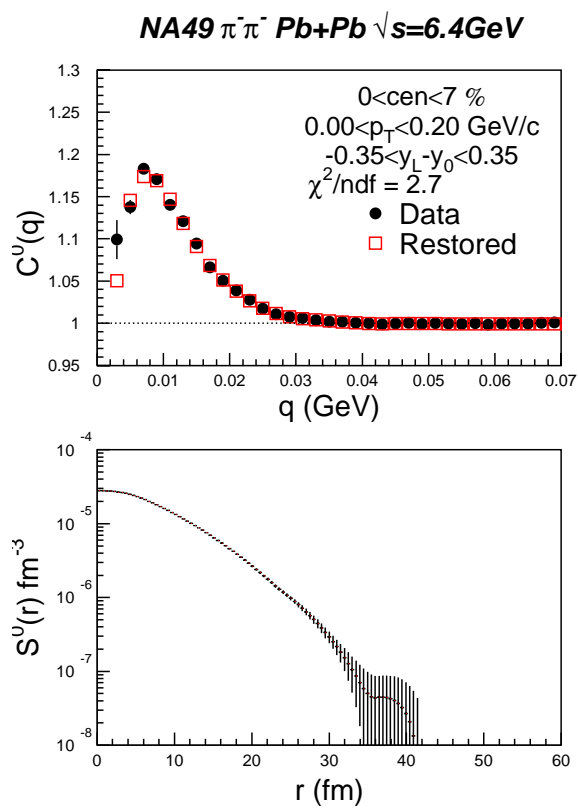

FIG. 9: (Color online) $\mathrm{l}=0$ moment $C^{0}(q)=R^{0}(q)+1$ (solid circles) and imaged source moment $S^{0}(r)$ for mid-rapidity low $p_{T} \pi^{-} \pi^{-}$ pairs from $20 \mathrm{AGeV}$ central $\mathrm{Pb}+\mathrm{Pb}$ collisions. Open squares in the top panel represent the correlation moment restored from the imaged source moment $S^{0}(r)$.
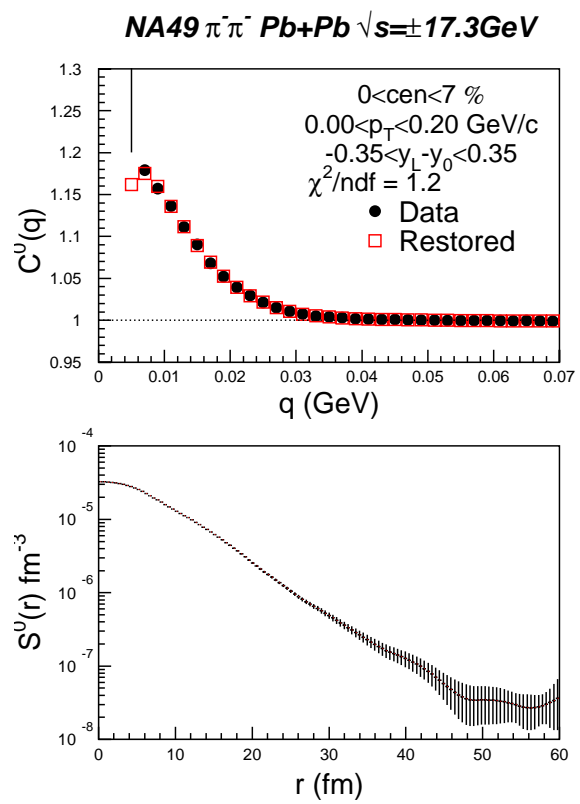

FIG. 10: (Color online) Same as Fig. 9 but for $158 \mathrm{AGeV}$ central $\mathrm{Pb}+\mathrm{Pb}$ collisions.

for $r>20 \mathrm{fm}$ is related to the difference in $C^{0}$ for $q<10 \mathrm{MeV}$.

Figs. 11 and 12 show that the negative contribution of the $l=2$ correlation moment $R_{x x}^{2}$ results in a positive contribution of the $l=2$ source moment $S_{x x}^{2}$. The negative contribution of $R_{x x}^{2}$ to $R^{0}$ causes the overall correlation function to be narrower in the $x$ direction compared to the angle-average correlation 

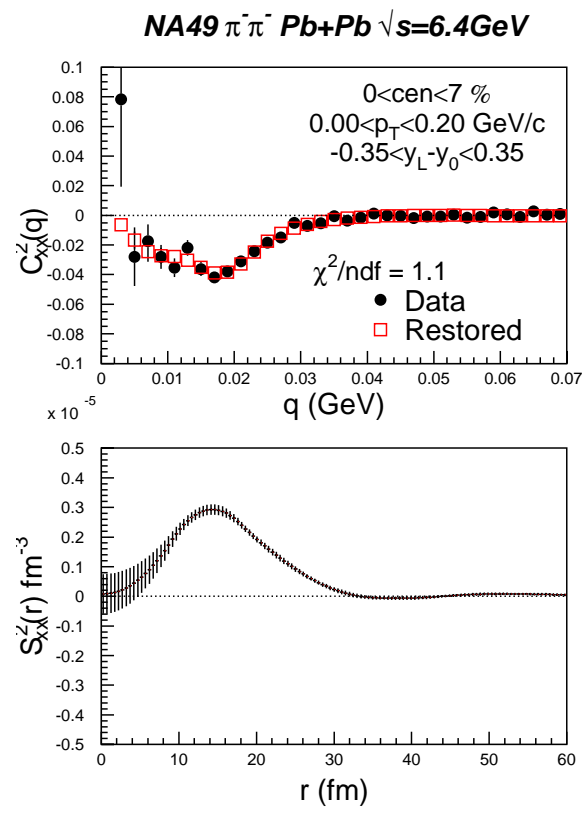

FIG. 11: (Color online) l=2 moment $C_{x x}^{2}(q)=R_{x x}^{2}(q)$ (solid circles) and the imaged source moment $S_{x x}^{2}(r)$ for mid-rapidity low $p_{T} \pi^{-} \pi^{-}$ pairs from $20 \mathrm{AGeV}$ central $\mathrm{Pb}+\mathrm{Pb}$ collisions. Open squares in the top panel represent the correlation moment restored from the imaged source moment $S_{x x}^{2}(r)$.

\section{$N A 49 \pi^{-} \pi^{-} P b+P b \sqrt{ }= \pm 17.3 G e V$}
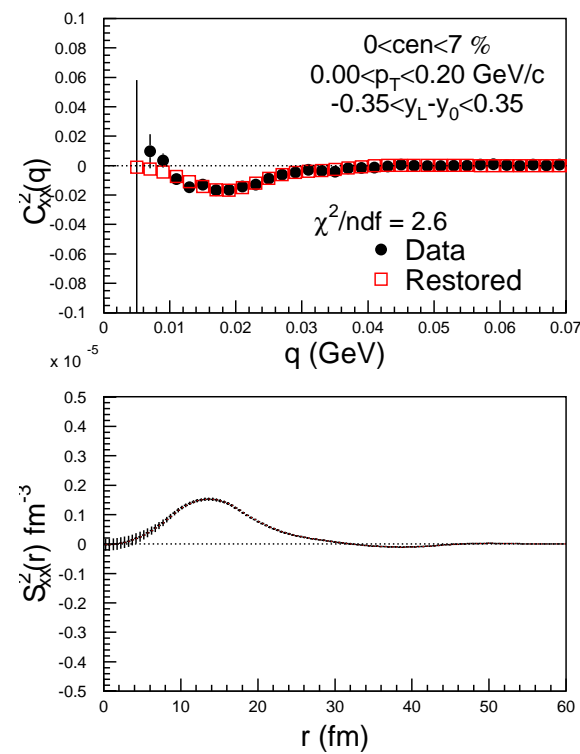

FIG. 12: (Color online) Same as Fig. 11 but for $158 \mathrm{AGeV}$ central $\mathrm{Pb}+\mathrm{Pb}$ collisions.

function. Consequently, the source function is broader in $x$ compared to the angle-averaged source function, hence the positive contribution of $S_{x x}^{2}$ to $S^{0}$.

The opposite effect takes place in the $y$ direction as shown by Figs. 13 and 14. The positive contribution to the $l=2$ correlation moment $R_{y y}^{2}$ results from a negative contribution of the $l=2$ source moment $S_{y y}^{2}$. The positive contribution of $R_{y y}^{2}$ causes the overall correlation function to be broader in the $y$ direction compared to the angle-average correlation function. Consequently, the source function is narrower in $y$ compared to the angle-average source function, hence the negative contribution of $S_{y y}^{2}$ to $S^{0}$.
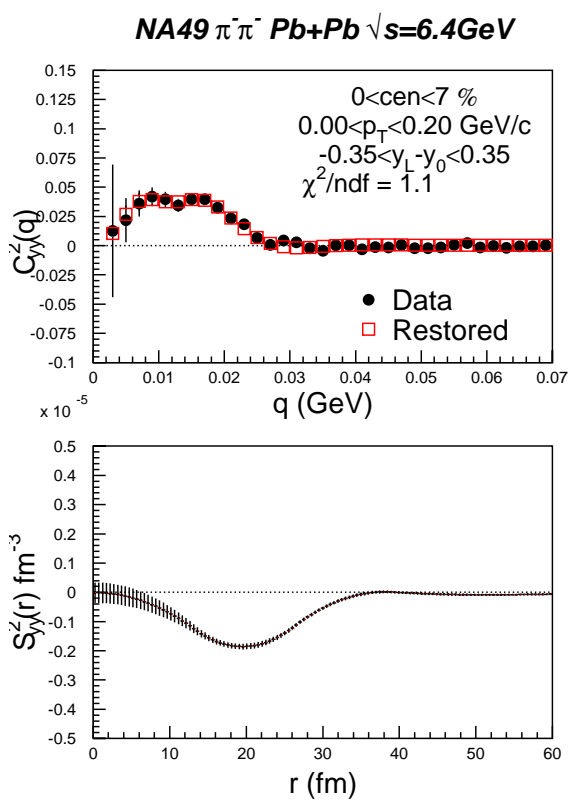

FIG. 13: (Color online) $1=2$ moment $C_{y y}^{2}(q)=R_{y y}^{2}(q)$ (solid circles) and the imaged source moment $S_{y y}^{2}(r)$ for mid-rapidity low $p_{T} \pi^{-} \pi^{-}$ pairs from $20 \mathrm{AGeV}$ central $\mathrm{Pb}+\mathrm{Pb}$ collisions. Open squares in the top panel represent the correlation moment restored from the imaged source moment $S_{y y}^{2}(r)$.

\section{RESULTS}

For the low $p_{T}$ pairs considered, the only significant source moments are found for multipolarities $l=0$ and 2. Hence, the net source functions in the $\mathrm{x}, \mathrm{y}$ and $\mathrm{z}$ directions are simply given by the sum of the 1D source $S^{0}$ and the corresponding $l=2$ moments $S_{i i}^{2}$ where $\mathrm{i}=\mathrm{x}$, y or z. Fig. 15 shows the total source function for mid-rapidity, low $p_{T}$ pion pairs from central $\mathrm{Pb}+\mathrm{Pb}$ collisions at $20 \mathrm{AGeV}$ (i.e $\sqrt{s}=6.4 \mathrm{AGeV}$ ). The source image is shown by squares while the triangles represent the ellipsoid (3D Gaussian) shape fit to the 3D correlation function. The source image and the Gaussian fit display good agreement in the $\mathrm{x}$ and $\mathrm{y}$ directions while the source image in the $\mathrm{z}$ direction exhibits a non-Gaussian tail for $r>20 \mathrm{fm}$.

The same conclusion is reached from Figs. 16 and 17 for low $p_{T}$ pion pairs from central $\mathrm{Pb}+\mathrm{Pb}$ collisions at $40 \mathrm{AGeV}$ $(\sqrt{s}=8.9 \mathrm{AGeV})$ and $80 \mathrm{AGeV}(\sqrt{s}=12.4 \mathrm{AGeV})$ respectively. In fact, the source image does not seem to change appreciably from $20 \mathrm{AGeV}$ to $80 \mathrm{AGeV}$, although the Gaussian fit in the $\mathrm{z}$ direction seems to broaden with beam energy. 

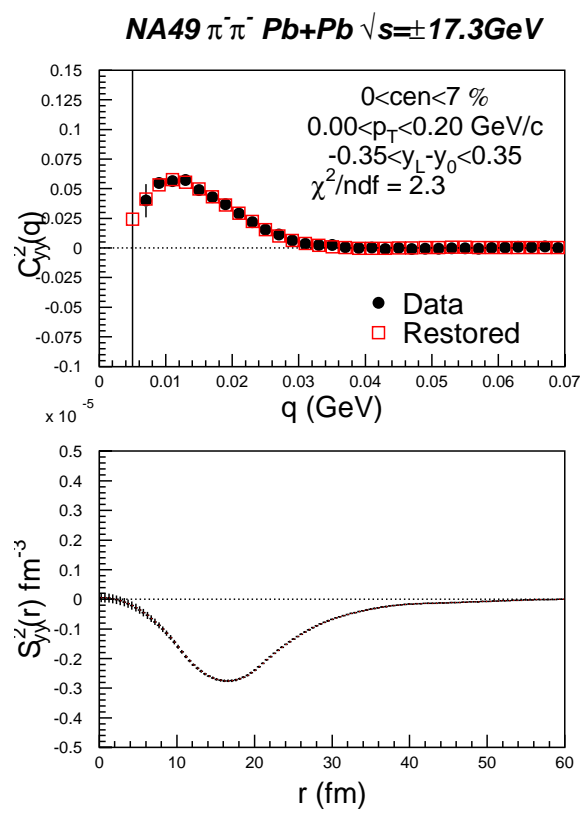

FIG. 14: (Color online) Same as Fig. 13 but for $158 \mathrm{AGeV}$ central $\mathrm{Pb}+\mathrm{Pb}$ collisions.

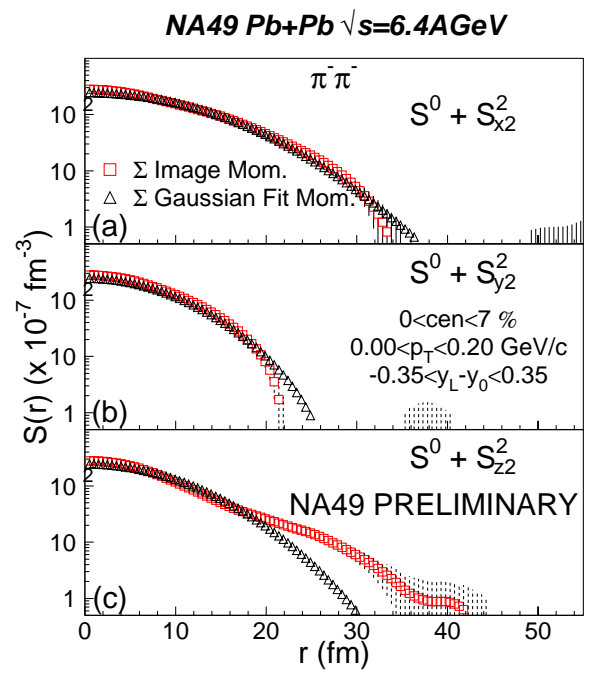

FIG. 15: (Color online) Total source function in (a) $\mathrm{x}$ (out) (b) $\mathrm{y}$ (side) (c) $\mathrm{z}$ (long) direction for low $p_{T}$ mid-rapidity $\pi^{-} \pi^{-}$pairs from central $\mathrm{Pb}+\mathrm{Pb}$ collisions at $\sqrt{s}=6.4 \mathrm{AGeV}$ (incident beam energy of $20 \mathrm{AGeV}$ ). Squares represent the source image while triangles represent the Gaussian assumption fit function result.

However, at $158 \mathrm{AGeV}$ incident energy $(\sqrt{s}=17.3 \mathrm{AGeV})$, Fig. 18 panels (a) and (c) show that there are significant deviations of the source image from the Gaussian fit in the $\mathrm{x}$ and $\mathrm{z}$ directions respectively: the source image seems to exhibit a non-Gaussian tail which extends beyond the range of the Gaussian fit for $r>17 \mathrm{fm}$ in the out and long directions.

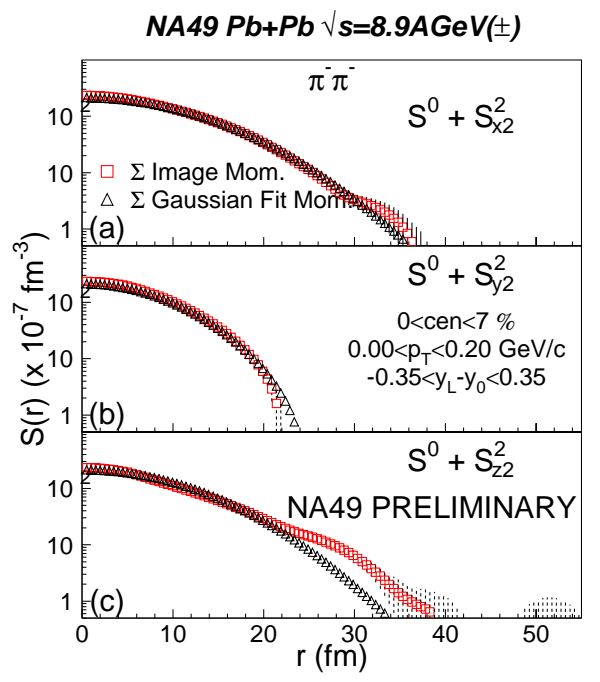

FIG. 16: (Color online) Same as Fig. 15 but for $\sqrt{s}=8.9 \mathrm{AGeV}$ (incident beam energy of $40 \mathrm{AGeV}$ ). The result is the average over runs with $+v e$ and -ve magnetic field.

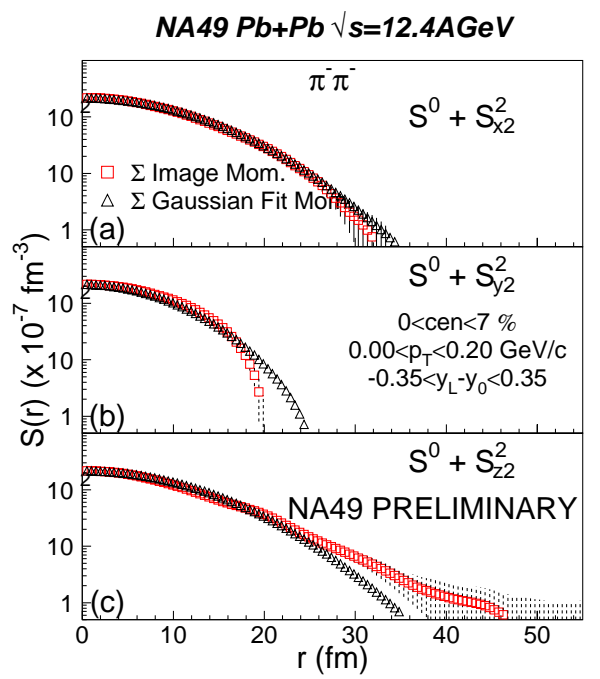

FIG. 17: (Color online) Same as Fig. 15 but for $\sqrt{s}=12.4 \mathrm{AGeV}$ (incident beam energy of $80 \mathrm{AGeV}$ ).

\section{DISCUSSION}

The evolution of the two-pion source image with increasing beam energy indicates that the source function is essentially Gaussian, albeit with some non-Gaussian tail in the long direction, and does not change appreciably from $20 \mathrm{AGeV}$ to $80 \mathrm{AGeV}$. However, it displays very prominent non-Gaussian tails at $158 \mathrm{AGeV}$ in the out and long directions. Hence, the long range structure, first observed at RHIC 


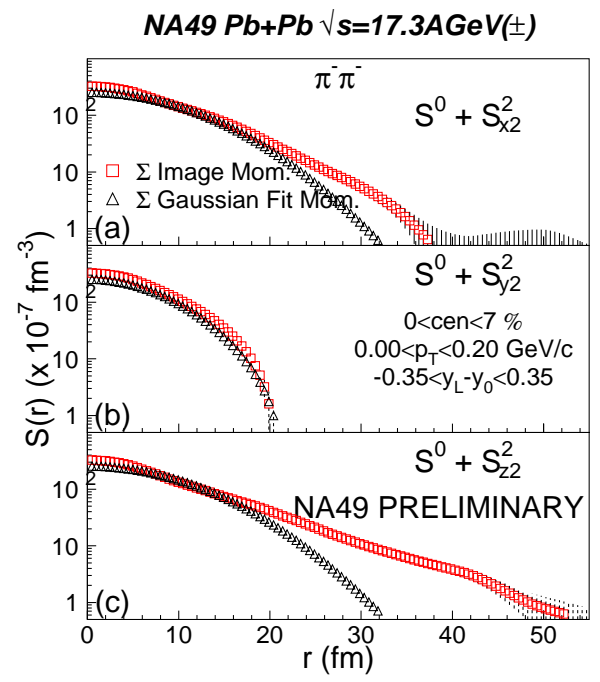

FIG. 18: (Color online) Same as Fig. 15 but for $\sqrt{s}=17.3 \mathrm{AGeV}$ (incident beam energy of $158 \mathrm{AGeV}$ ). The result is the average over runs with +ve and -ve magnetic field. $(\sqrt{s}=200 \mathrm{AGeV})[6]$, is already manifest at much lower energies at the SPS. This points to the expectation that there are new physics processes which set in between $80 \mathrm{AGeV}$ $(\sqrt{s}=12.4 \mathrm{AGeV})$ and $158 \mathrm{AGeV}(\sqrt{s}=17.3 \mathrm{AGeV})$ which result in the non-Gaussian tails in this energy regime.

Could the non-Gaussian tails observed at $158 \mathrm{AGeV}$ be due to resonance decays such as the $\omega$ meson ? However, the general finding is that the resonance population does not change significantly from $\sqrt{s}=12.4 \mathrm{AGeV}$ to $\sqrt{s}=17.3 \mathrm{AGeV}$. Hence, it is unlikely that resonance decays could account for the observed non-Gaussian tail at $158 \mathrm{AGeV}$.

\section{CONCLUSIONS}

The NA49 Collaboration has performed the first extraction of emission source functions for mid-rapidity, low $p_{T}$ pions produced in central $\mathrm{Pb}+\mathrm{Pb}$ collisions over the beam energy range 20-158 AGeV at SPS. The source functions are essentially Gaussian and change little from 20 to $80 \mathrm{AGeV}$. At $158 \mathrm{AGeV}$, the two-pion source function displays nonGaussian tails in the out and long directions. It is unlikely that these non-Gaussian tails originate from secondary pions produced in resonance decays.
[1] Quark Matter 2005 Proceedings of the 18th International Conference on Ultra-Relativistic Nucleus-Nucleus Collisions, Budapest, Hungary, August 4-9, 2005, Nucl. Phys. A 774 (2006).

[2] D.A. Brown and P. Danielewicz, Phys. Lett. B 398, 252 (1997).

[3] D.A. Brown and P. Danielewicz, Phys. Rev. C 57, 2474 (1998).

[4] D.A. Brown and P. Danielewicz, Phys. Rev. C 64, 14902 (2001).

[5] P. Chung et al, Nucl. Phys. A 749, 275c (2005).

[6] S.S. Adler et al.(PHENIX Collaboration), nucl-ex/0605032.

[7] C. Alt et al.(NA49 Collaboration), CERN-SPSC-2005-041, CERN-SPSC-P-264-ADD-12, Nov 2005.
[8] S. Afanasiev et al., Nucl. Instrum. Meth. A 430, 210 (1999).

[9] P. Danielewicz and S. Pratt, Phys. Lett. B 618, 60 (2005).

[10] P. Chung et al, XXXV International Symposium on Multiparticle Dynamics, Kromeriz, Czech Republic, 9-15 August 2005, and Workshop on Particle Correlations and Femtoscopy, 15-17 August 2005, p. 499 (2006).

[11] S.E. Koonin, Phys. Lett. B 70, 43 (1977); S. Pratt, T. Csörgö, and T. Zimányi, Phys. Rev. C 42, 2646 (1990). 